NASA-CR-204601

\title{
SHORT-TERM CHROMOSPHERIC VARIABILITY IN $\propto$ TAURI (K5 III): $0 \therefore-445$ RESULTS FROM IUE TIME SERIES OBSERVATIONS
}

\author{
MANFRED CUNTZ ${ }^{1,2}$ \\ Joint Institute for Laboratory Astrophysics, University of Colorado and National Institute of Standards and Technology, \\ Campus Box 440, Boulder, CO 80309-0440; cuntz@jila.colorado.edu \\ Bryan D. Deeney and Alexander Brown \\ Center for Astrophysics and Space Astronomy, Campus Box 389, University of Colorado, Boulder, CO 80309-0389; \\ deeneyb $@$ echidna.colorado.edu, ab@echidna.colorado.edu \\ AND \\ Robert E. STENCEL \\ Department of Physics and Astronomy, University of Denver, Denver, CO 80208; rstencel $(a$ du.edu \\ Received 1995 September 28; accepted 1995 December 20
}

\begin{abstract}
We evaluate time series observations of chromospheric lines ( $\mathrm{Mg} \mathrm{II}, \mathrm{Mg} \mathrm{I}$, and $\mathrm{C}$ II) for the $\mathrm{K}$ giant $\alpha$ Tau obtained using the IUE LWP camera at high dispersion. These observations cover a time span of about 2 weeks in 1994 February-March and were designed to resolve variations occurring within hours, days, and weeks. We consider the observational results in relation to theoretical acoustic heating models, motivated by the fact that $\alpha$ Tau may exhibit a basal (i.e., minimum) level of chromospheric activity. The data reveal flux variations between the extremes of $8 \%$ in $\mathrm{Mg}$ II $h+k$ and $15 \%$ in each emission component. These variations occur on timescales as short as $8 \mathrm{hr}$ but not on timescales longer than $\sim 3$ days. For the $h$ and $k$ components, flux variations occurring on a timescale as short as $1.5 \mathrm{hr}$ are also found. These changes are often not correlated (and are sometimes even anticorrelated), leading to remarkable differences in the $h / k$ ratios. We argue that these results are consistent with the presence of strong acoustic shocks, which can lead to variable $\mathrm{Mg}$ II line emission when only a small number of strong shocks are propagating through the atmosphere. We deduce the electron density in the $\mathrm{C}$ II] $\lambda 2325$ line formation region to be $\log N_{e} \simeq 9.0$, in agreement with previous studies. Our data provide evidence that the $\mathrm{Mg}$ II basal flux limit for $\mathrm{K}$ giants might be a factor of 4 higher than suggested by Rutten et al.

Subject headings: stars: activity - stars: chromospheres - stars: individual ( $\alpha$ Tauri) stars: late-type - ultraviolet: stars
\end{abstract}

\section{INTRODUCTION}

The outer atmospheres of $\mathrm{K}$ giant stars have been studied extensively with satellite-based instruments over the past two decades. Many of these stars are considered to be "inactive" as they have relatively low chromospheric emission losses and do not possess detectable coronae (e.g., Ayres, Fleming, \& Schmitt 1991; Ayres et al. 1995). For $\alpha$ Tauri (Aldebaran $=$ HD $29139=$ HR 1457; K 5 III), important information about the thermodynamic structure of the outer atmosphere has been obtained from IUE (Kelch et al. 1978; Judge 1986) and Hubble Space Telescope (HST) Goddard High Resolution Spectrograph (GHRS) data (Carpenter et al. 1991). It has been found that the outer atmospheres of $\alpha$ Tau and other inactive $\mathrm{K}$ giants are dominated by chromospheres with temperatures between 5000 and $15,000 \mathrm{~K}$, perhaps accompanied by tiny layers at somewhat higher temperatures. Stellar winds with moderately large mass-loss rates, of order $10^{-9} M_{\odot} \mathrm{yr}^{-1}$ (or somewhat below), are also present. The final flow speeds of the winds are relatively low, probably not exceeding $50 \mathrm{~km} \mathrm{~s}^{-1}$. Judge \& Stencel (1991) have evaluated these findings and have discussed links to the evolutionary status of the stars by interpreting the thermodynamic properties of the atmospheres and winds by means of statistical concepts. None-

\footnotetext{
${ }^{1}$ Also at Institut für Theoretische Astrophysik, Universität Heidelberg, Tiergartenstraße 15, D-69121 Heidelberg, Germany.

2 Present address: Center for Space Plasma and Aeronomic Research, University of Alabama in Huntsville, Huntsville, AL 35899.
}

theless, the underlying physical processes responsible for these properties remain only barely understood, partly owing to the lack of theoretical models and suitable chromospheric line diagnostics (see, e.g., Jordan \& Linsky 1987). Ideally, chromospheric line diagnostics can give observational insight into the height-dependent atmospheric energy and momentum input, which are key to the chromospheric phenomenon.

Why should we study the outer atmospheric dynamics of inactive $\mathrm{K}$ giant stars at all? One basic reason is that these stars represent the far distant future of solar-type activity. When solar-type stars evolve up the giant branch, their angular momentum subsides, in part because of the increase in the moment of inertia and magnetic braking resulting from the onset of massive stellar winds (Schrijver 1993; Charbonneau, Schrijver, \& MacGregor 1996). Most intriguingly, the stellar rotation periods of $\mathrm{K}$ giants are found to be extremely long, e.g., 223 days for $\alpha$ Boo (K 1.5 III) and 643 days for $\alpha$ Tau (Hatzes \& Cochran 1993). During the course of post-main-sequence evolution, the stellar magnetic fields are expected to weaken dramatically and are also likely to change their topology, which is found to be relevant to the outer atmospheric dynamics (Rosner et al. 1991, 1995). It has been argued that the dominant chromospheric heating mechanism in inactive late-type giants might be essentially acoustic rather than magnetic. This result has found support in studies by Schrijver (1987), Pasquioi, Pallavicini, \& Pakull (1988), Dupree, Hartmann, 

\& Smith (1990), Rutten et al. (1991), and Strassmeier et al. (1994), which show that most single noncoronal giants and supergiants have chromospheric fluxes, in agreement with or close to the empirically deduced basal flux limits, a suspected indicator of pure acoustic heating. Judge \& Stencel (1991) extrapolated the Mg II basal flux limits to low effective temperatures in order to evaluate the activity level of noncoronal $\mathrm{K}$ giant stars. These limits were successfully reproduced by detailed theoretical ab initio models based on acoustic energy dissipation (Cuntz, Rammacher, \& Ulmschneider 1994; Buchholz \& Ulmschneider 1994; Buchholz 1995). The agreements were found to be within a factor of 2 for stars of widely different spectral types and luminosity classes. On the other hand, evidence also exists indicating that even inactive noncoronal giants and supergiants might have some magnetic field coverage remaining. These magnetic fields may be important for explaining the observed level of atmospheric turbulence (Judge \& Cuntz 1993) and the stellar mass-loss behavior (Hartmann \& MacGregor 1980; Cuntz 1990b; Sutmann \& Cuntz 1995).

In order to interpret the chromospheric line emission in terms of physical parameters (e.g., energy and momentum balance of the chromosphere and wind), studies of line formation in low-density plasma are ultimately required. These studies yield empirical results concerning the formation of individual chromospheric lines. For $\alpha$ Tau, results were given by Stencel et al. (1981), Brown \& Carpenter (1984), Carpenter, Brown, \& Stencel (1985), Judge (1986), and Byrne et al. (1988). The empirical technique gives information about thermodynamic quantities present in the line formation region. Nonetheless, this technique is only of limited use as the lines in question are usually formed over a large range of mass column density (Judge 1990) and are furthermore influenced by time-dependent effects. Therefore, it is often unclear what the global measures of physical properties represent. A recent example demonstrating that highly resolved $\mathrm{C}$ II] line profiles can provide information about chromospheric substructure velocities has been given by Judge (1994), who reanalyzed the GHRS data for $\alpha$ Tau. Judge found significant evidence for multiple density components in this star moving inward and outward with respect to the stellar surface.

Other studies of stellar outer atmospheres involve the calculation of semiempirical models. These models give information about the run of the velocity and the various thermodynamic quantities in the atmosphere, which are assumed to be smooth functions of the atmospheric height. These functions are varied in order to fit observed line strengths and profiles. In the case of $\alpha$ Tau, Kelch et al. (1978) found evidence that the atmospheric temperature rises from 2700 to $12,000 \mathrm{~K}$ between $3.0 \times 10^{-1}$ and $9.5 \times 10^{-5} \mathrm{~g} \mathrm{~cm}^{-2}$ mass column density. However, the relevance of these models is seriously in question because the lines studied (e.g., $\mathrm{Ca}$ II $\mathrm{H}+\mathrm{K}$ ) are often formed in a highly inhomogeneous environment that contains an irregular distribution of high-temperature regions. In this case, the chromospheric line formation cannot be assumed to be associated with a time-independent "mean" atmosphere. It is indeed possible that the time-averaged temperature decreases with increasing atmospheric height (or decreasing mass column density), despite the fact that the atmosphere contains a significant number of high-temperature spikes (see, e.g., Carlsson \& Stein 1995), which are prominently responsible for reproducing the observed $\mathrm{Mg}$ II and $\mathrm{Ca}$ II emission. Detailed observations of $\alpha$ Tau and other giants show that the atmospheres also contain plasma at both low and high temperature, as revealed by observations of the fundamental rotation-vibration band of $\mathrm{CO}$ at $4.6 \mu \mathrm{m}$ (Wiedemann et al. 1994) and variable absorption and emission in He I $\lambda 10830$ (O'Brien \& Lambert 1986). Unified models for these different types of inhomogeneities are, however, clearly beyond the scope of current cool star atmospheric models.

The work presented in this paper represents an attempt to constrain the degree of chromospheric variability and inhomogeneity in $\alpha$ Tau, as manifested in the variability patterns of various chromospheric emission lines. We compare the observed line variability with previous theoretical studies and evaluate the overall chromospheric activity level. In $\S 2$, we give details of the observations. Section 3 discusses the data reduction and analysis. In $\S 4$, we give results regarding our $\mathrm{Mg} \mathrm{II}, \mathrm{Mg}$ I, and $\mathrm{C}$ II observations. We also comment on existing theoretical models. Section 5 offers our conclusions.

\section{OBSERVATIONS}

On 12 days, between 1994 February 25 and March 11, x Tau was observed with the IUE LWP camera in high dispersion. We accumulated a total of 50 images over 6 US 1 and 8.5 US 2 shifts. The images are divided into three categories according to exposure time. We obtained 23 short exposures of 2.5-3 minutes (depending on the suspected radiation level) to get information about variations in the $\mathrm{Mg}$ II line cores and to measure the $\mathrm{Mg}$ II emission fluxes. We also obtained 24 images of moderate exposure length (10-14 minutes) to search for possible changes in the $\mathrm{Mg}$ II $h$ and $k$ line wings. The cores of the $\mathbf{M g}$ II lines in these images are overexposed, as expected. Finally, three images with 90 minute exposure times were taken in order to properly expose the $\mathrm{C}$ II] 22325 multiplet and the $\mathrm{Mg}$ I 22852 line, which are a factor of 20 weaker than the $\mathrm{Mg}$ II lines. The $\mathrm{C}$ II] multiplet is particularly useful for obtaining information about the chromospheric electron density.

\section{DATA REDUCTION AND ANALYSIS}

The spectra were reduced interactively at the University of Colorado, Boulder, from the standard IUE Spectral Image Processing System (SIPS) files provided to the Guest Observer. All images were processed with the new LWP intensity transfer function (LWP ITF2), described by Cassatella, Lloyd, \& Gonzales Riestra (1988). The absolute flux calibration was determined using the LWP ITF2 inverse sensitivity function $S_{\lambda}^{-1}$ and the "new" high-dispersion calibration function $C(\lambda)$ (Cassatella et al. 1990). The fluxes were corrected both for short-term variations in sensitivity caused by changes in camera temperature using the IUE SIPS routine TEMPCOR, and for the long-term sensitivity degradation of the LWP camera by using LWP images obtained after epoch 1984.5 (Garhart 1992).

We searched for possible variability in the fluxes of the $\mathrm{Mg}$ II $h$ and $k$ resonance lines, using only the short-exposure LWP spectra in which the $\mathrm{Mg}$ II emission features were unsaturated. These observations were spread unevenly over 2 weeks in an attempt to detect variations on timescales ranging from hours to weeks. Considerable care must be taken in analyzing the $\mathrm{Mg}$ II features if small-scale fluctuations are to be detected. Following Ayres et al. (1995), we minimized the influence of subjective errors on our flux 
measurements by registering the $\mathrm{Mg}$ II emission profiles to a common wavelength scale, then integrating the flux over a fixed bandpass. The profiles were brought to the common wavelength scale through an iterative process. First, the initial line profiles were co-added to form a mean profile. The individual profiles then were cross-correlated against the mean, in order to establish the offset of each profile from the reference. In order to avoid interpolation between wavelength bins, and the spectral smoothing that results, the profiles were shifted by integral numbers of bins. The shifted profiles were used to compute a new mean profile, and the entire process was repeated until the residual shifts converged to zero.

The integration bandpass was chosen by a visual examination of the composite profile, constructed by co-adding the registered profiles. The bandpass was then applied uniformly to the individual registered profiles to derive the flux measurements summarized in Table 1 . We derived the $1 \sigma$ error estimates given in Table 1 by assuming that the photometric noise appropriate to typical LW-HI exposures obeys a simple two-component distribution: $\sigma \approx \sigma_{0}$ $+\sigma_{1}\left(I_{\lambda} / I_{\max }\right)^{1 / 2}$. The first term refers to the often dominant component of the noise that is independent of the local intensity and that arises from the background of the Vidicon image contributed partly by the camera pedestal and partly by radiation "fogging." The second term refers to the component of the photometric error that depends on the intensity, analogous to the behavior of a photoncounting sensor with negligible background. We estimated the two coefficients empirically in each exposure by examining the rms deviation of the profile fluxes from those of a reference line shape scaled to the same integrated flux. This approach assumes that the shape of the line does not change. In the presence of significant morphological profile variations, the method overestimates the photometric noise. In the empirical "calibration" of the noise model, we compensated for any prior smoothing of the oversampled pro- files and for the intrinsic spatial averaging properties of the detectors (see discussion by Ayres 1993).

\section{RESULTS AND DISCUSSION}

\section{1. $\mathrm{Mg} \mathrm{II}$}

The $\mathrm{Mg}$ II $h$ and $k$ lines are particularly useful because they are easily accessible diagnostics that have sufficient opacity over a wide temperature range $\left(4000 \mathrm{~K} \lesssim T_{e} \lesssim\right.$ $8000 \mathrm{~K}$ ) to reveal detailed information on conditions, from the low chromosphere through to the outermost detectable parts of the wind. The Mg II $h, k$, and $h+k$ fluxes, as well as the $h / k$ ratios of $\alpha$ Tau from the 23 short-exposure images, are presented in Figure 1. Figure 2 shows, in more detail, the series of observations made between JD $(+2,440,000)$ 9415.5 and 9417.5 , which has particularly narrow time spacing.

The data give unique insight into the variability of the $\mathrm{Mg}$ II chromospheric emission of $\alpha$ Tau. The mean fluxes measured for the $\mathrm{Mg}$ II lines are $F_{h}=7.37 \times 10^{-11} \mathrm{ergs}$ $\mathrm{cm}^{-2} \mathrm{~s}^{-1}$ and $F_{k}=11.38 \times 10^{-11} \mathrm{ergs} \mathrm{cm}^{-2} \mathrm{~s}^{-1}$. Between the extremes, the flux varies by $15 \%$ in each emission component and by $8 \%$ in $\mathrm{Mg}$ II $h+k$. The variations are found to occur on timescales as short as $8 \mathrm{hr}$, but not on significantly longer timescales such as several days or weeks. Most surprisingly, it is found that the changes in $\mathrm{Mg}$ II $h$ and $k$ are often not correlated and, at times, appear to be anticorrelated. A chromospheric brightening caused by the passage of a shock should appear first in the $\mathrm{Mg}$ II $h$ line, which, being a factor of 2 less optically thick than the $k$ line, is sensitive to conditions deeper in the chromosphere. Flux variations observed in images obtained on JD 2,449,417 are at least consistent with such a scenario. On this day, an increase of $6 \%$ or more in the total $\mathrm{Mg}$ II flux has occurred, enduring at least $8.5-12 \mathrm{hr}$. This increase is clearly visible in both the $\mathrm{Mg}$ II $h$ and $k$ lines, but it is not possible to uniquely identify a temporal offset between the changes in TABLE 1

Mg II $\lambda 2800$ Emission Line FluXes of $\alpha$ TAuri

\begin{tabular}{|c|c|c|c|c|c|c|c|}
\hline \multirow{2}{*}{$\begin{array}{l}\text { IMAGE } \\
\text { (LWP) }\end{array}$} & \multirow{2}{*}{$\begin{array}{l}\text { DOY } \\
(1994)\end{array}$} & \multirow{2}{*}{$\begin{array}{c}\text { EXPOSURE } \\
\text { START } \\
\text { (UT) }\end{array}$} & \multirow{2}{*}{$\begin{array}{c}\text { EXPOSURE } \\
\text { TimE } \\
\text { (minutes) }\end{array}$} & \multirow[b]{2}{*}{ JD $2,440,000+$} & \multicolumn{2}{|c|}{ 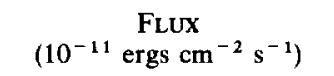 } & \multirow{2}{*}{$\begin{array}{c}\text { FLux RATIO } \\
h / k\end{array}$} \\
\hline & & & & & $h$ & $k$ & \\
\hline 27492 . & 55 & $23: 51$ & 2.5 & 9408.494 & $7.54(0.35)$ & $11.69(0.41)$ & $0.645(0.038)$ \\
\hline 27495. & 56 & $2: 26$ & 2.5 & 9408.602 & $7.30(0.11)$ & $11.34(0.24)$ & $0.643(0.017)$ \\
\hline $27505 .$. & 56 & $19: 51$ & 3.0 & 9409.327 & $7.77(0.10)$ & $11.39(0.28)$ & $0.682(0.019)$ \\
\hline $27510 \ldots$ & 57 & $0: 28$ & 2.5 & 9409.520 & $7.34(0.15)$ & $11.43(0.34)$ & $0.642(0.023)$ \\
\hline $27520 \ldots$ & 57 & $17: 53$ & 3.0 & 9410.245 & $7.47(0.29)$ & $10.88(0.36)$ & $0.687(0.035)$ \\
\hline 27526 .. & 57 & $23: 30$ & 3.0 & 9410.479 & $7.63(0.36)$ & $11.17(0.35)$ & $0.683(0.039)$ \\
\hline 27557. & 62 & $23: 24$ & 3.0 & 9415.475 & $7.24(0.11)$ & $11.44(0.18)$ & $0.633(0.014)$ \\
\hline 27565 .. & 63 & $14: 48$ & 3.0 & 9416.116 & $7.04(0.17)$ & $11.29(0.33)$ & $0.623(0.024)$ \\
\hline $27577 \ldots$ & 64 & $1: 38$ & 3.0 & 9416.568 & $7.53(0.21)$ & $11.10(0.25)$ & $0.678(0.024)$ \\
\hline $27580 \ldots$ & 64 & $11: 58$ & 3.0 & 9416.999 & $6.91(0.10)$ & $11.45(0.19)$ & $0.604(0.013)$ \\
\hline $27583 \ldots$ & 64 & $15: 49$ & 3.0 & 9417.159 & $7.53(0.09)$ & $11.18(0.15)$ & $0.674(0.012)$ \\
\hline $27585 \ldots \ldots$ & 64 & $17: 17$ & 3.0 & 9417.220 & $7.35(0.11)$ & $11.36(0.13)$ & $0.647(0.012)$ \\
\hline $27587 \ldots \ldots$ & 64 & $18: 38$ & 3.0 & 9417.276 & $7.25(0.20)$ & $11.77(0.10)$ & $0.616(0.018)$ \\
\hline $27589 \ldots$ & 64 & $20: 03$ & 3.0 & 9417.335 & $7.44(0.12)$ & $11.66(0.14)$ & $0.638(0.013)$ \\
\hline 27591 .. & 64 & $21: 29$ & 3.0 & 9417.396 & $7.95(0.09)$ & $11.39(0.10)$ & $0.698(0.010)$ \\
\hline $27593 \ldots$ & 64 & $22: 53$ & 3.0 & 9417.453 & $7.46(0.08)$ & $11.66(0.17)$ & $0.640(0.011)$ \\
\hline $27595 \ldots$ & 65 & $0: 16$ & 3.0 & 9417.511 & $7.67(0.08)$ & $11.87(0.14)$ & $0.646(0.010)$ \\
\hline $27615 \ldots \ldots$ & 67 & $11: 58$ & 3.0 & 9419.999 & $7.27(0.09)$ & $11.41(0.13)$ & $0.637(0.011)$ \\
\hline $27622 \ldots \ldots$ & 67 & $19: 57$ & 3.0 & 9420.331 & $6.84(0.11)$ & $11.16(0.16)$ & $0.613(0.013)$ \\
\hline $27632 \ldots \ldots$ & 68 & $20: 11$ & 3.0 & 9421.341 & $7.18(0.15)$ & $11.29(0.12)$ & $0.636(0.015)$ \\
\hline $27638 \ldots \ldots$ & 69 & $1: 47$ & 3.0 & 9421.574 & $7.16(0.13)$ & $11.27(0.17)$ & $0.636(0.015)$ \\
\hline $27645 \ldots \ldots$ & 69 & $20: 28$ & 3.0 & 9422.353 & $7.37(0.19)$ & $11.49(0.16)$ & $0.641(0.019)$ \\
\hline $27650 \ldots \ldots$ & 70 & $1: 04$ & 3.0 & 9422.545 & $7.33(0.12)$ & $11.15(0.23)$ & $0.658(0.017)$ \\
\hline
\end{tabular}

NoTE.--The numbers in parenthesis indicate $1 \sigma$ error estimates. 


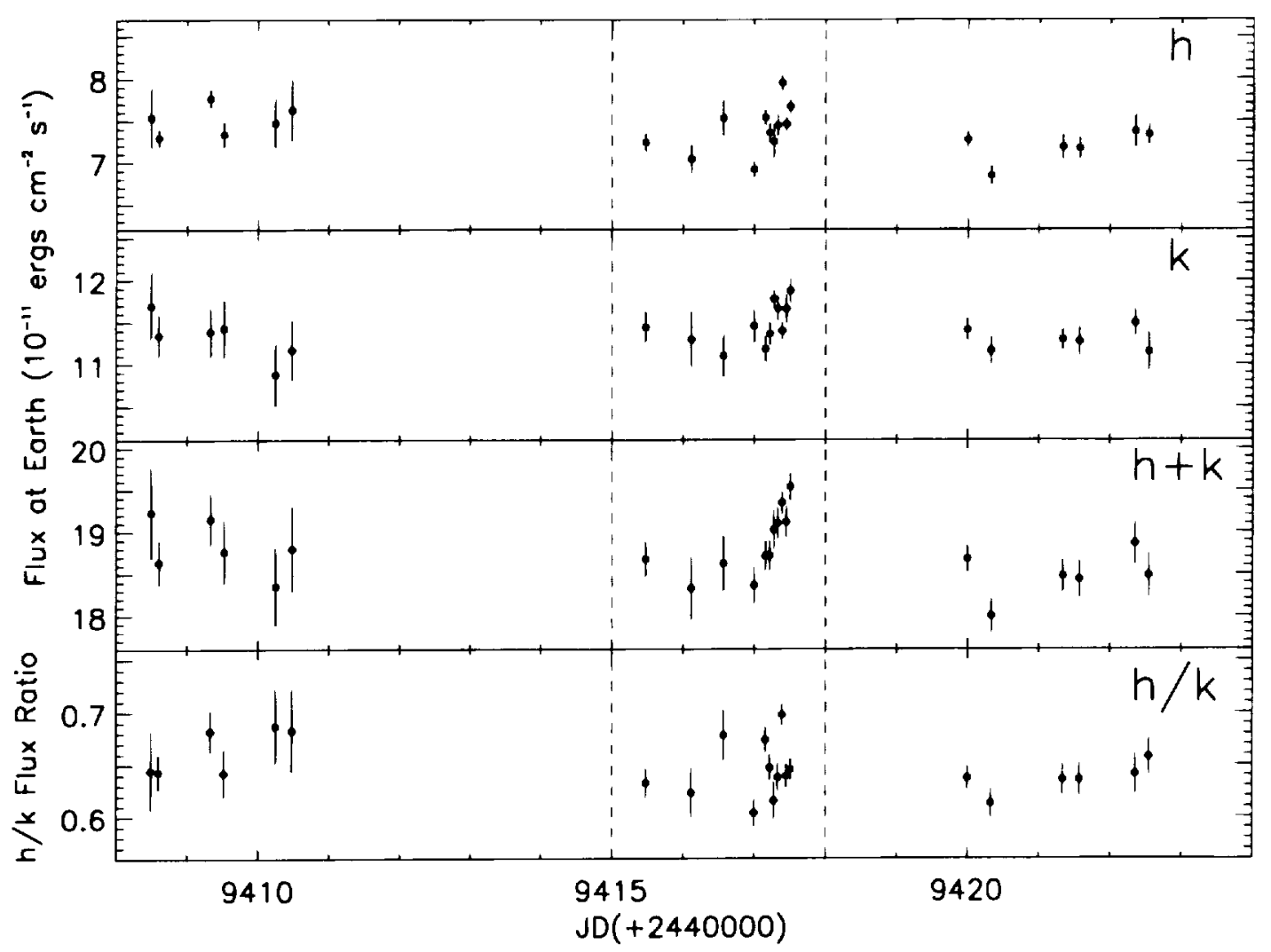

FIG. 1.-Integrated Mg II fluxes obtained during 2 weeks of $I U E$ observations in 1994 February-March. Panel shows the Mg II $h, k, h+k$ fluxes, and the $h / k$ flux ratios. Error bars represent $1 \sigma$ standard deviations. Note the extremely nonequidistant spacing of the data. The subset of data displayed in Fig. 2 is indicated by the window bordered by dashed lines.

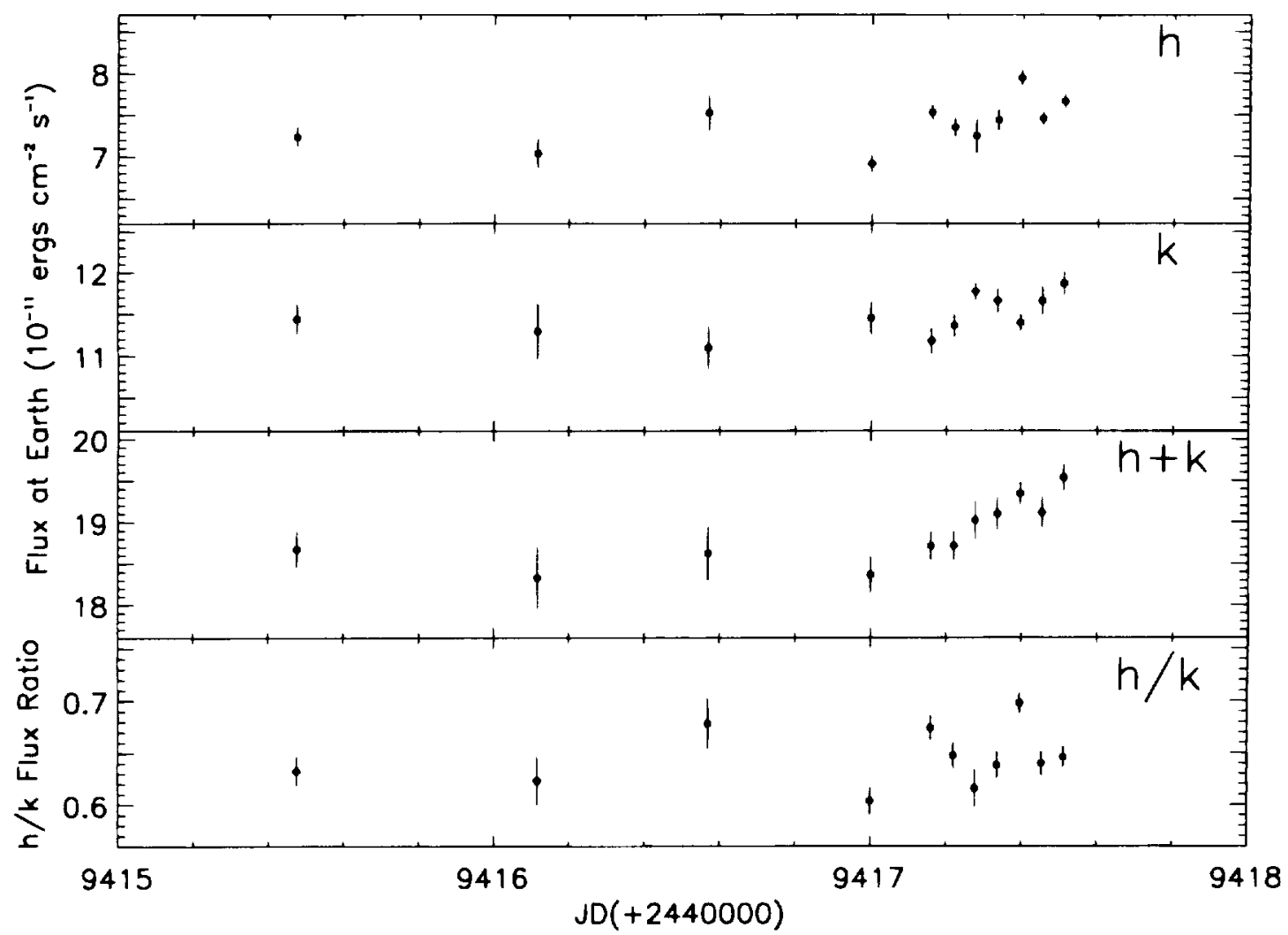

FIG. 2.-Same as Fig. 1, but now for a subset of the data obtained during a 2 day time span 
these components. In fact, they show uncorrelated flux variations with a timescale of $1.5 \mathrm{hr}$ or less. Also, the duration of this $\mathrm{Mg}$ II flux increase cannot be precisely determined. Because of the data gaps, it can only be estimated that this increased emission might persist over a time span between 0.5 and 3 days. Figure 3 shows that the flux increase is confined to the $\mathrm{Mg}$ II line cores, which form in the outermost parts of the line formation region.

We also found another instance of $\mathrm{Mg}$ II $h+k$ flux increase occurring between JD 2,449,420 and JD 2,449,422. During that time, a $\mathrm{Mg}$ II flux increase of about $5 \%$ occurs. Because of the lack of data points, however, this increase is less significant than that found on JD 2,449,417. In fact, it is possible that the increase persisted only $24 \mathrm{hr}$ instead of 48 $\mathrm{hr}$. This increase is preceded by a rapid decrease in the $\mathrm{Mg}$ II $h+k$ flux of $4 \%$ occurring over a time span of $8 \mathrm{hr}$. This change is particularly well pronounced in the $\mathrm{Mg}$ II $h$ line.

We searched for variations in the $\mathrm{Mg}$ II $h$ and $k$ line wings, using the 24 moderate-length exposures obtained concurrently with the shorter exposures previously discussed. We visually examined the overplotted $\mathrm{Mg}$ II profiles from all 24 images but were unable to find any significant changes in the $\mathrm{Mg}$ II line wings. We attribute this result to radiative transfer effects. Since photons in the line core have first to migrate to the line wings in order to escape, they are subjected to multiple scattering. The scatterings will average out local variations in the line flux due to the presence of different thermodynamic conditions deep within the chromosphere.

A further issue is the overall $\mathrm{Mg}$ II activity level. Assuming an angular diameter for $\alpha$ Tau of 20.2 mas (Di Benedetto \& Rabbia 1987), our minimum Mg II $h+k$ flux corresponds to a minimum surface flux of $7.7 \times 10^{4}$ ergs $\mathrm{cm}^{-2} \mathrm{~s}^{-1}$. This value for the surface flux agrees to within $10 \%$ of that found by Judge (1986). If $\alpha$ Tau is indeed a "chromospheric basal flux star" (see $§ 1$ ), these observations seem to indicate that the basal $\mathrm{Mg}$ II emission flux for $\mathrm{K}$ giants is about a factor of 4 higher than suggested by Rutten et al. (1991).

\section{2. $\mathrm{Mg} I$ and $\mathrm{C}$ II}

The $\mathrm{Mg}$ I $\lambda 2852$ and $\mathrm{C}$ II] $\lambda 2325$ profiles obtained in the three 90 minute exposures are shown in Figures 4 and 5 , respectively. The Julian dates $(+2,440,000)$ at the beginning of the observations were $9416.007,9417.056$, and 9420.057 . As discussed by Stencel et al. (1981), the intensities of the $\mathrm{C}$ II intersystem multiplet near $2325 \AA$ depend on the electron density $N_{e}$ in the range $10^{9}<N_{e}<10^{7}$ and may be used as electron density diagnostics in cool giants and supergiants. Ideally, we wish to compute the intensity ratios $R_{1}\left(=F_{2325.38} / F_{2328.10}\right), R_{2}\left(=F_{2325.38} / F_{2326.91}\right)$, and $R_{3}\left(=F_{2324.67} / F_{2326.91}\right)$, where $F_{\lambda(\dot{A})}$ is the integrated flux of the $\mathrm{C}$ II] line with laboratory wavelength $\lambda$. Each intensity ratio leads to a separate estimate of the electron density for each image. Unfortunately, the $\mathrm{C}$ II] line at $2326.91 \AA$ is blended with the Fe II line at $2327.40 \AA$ (Judge \& Jordan 1991), making the line ratios $R_{2}$ and $R_{3}$ difficult to determine. This problem can only be alleviated with the use of high-quality data, as given by HST GHRS (Carpenter et al. 1991).

Therefore, we have calculated only the line intensity ratio $R_{1}$. Using the atomic data of Lennon et al. (1985), we find that $\log N_{e}=9.2,9.0$, and 8.7 for our three images LWP 27564,27582 , and 27617 , respectively. The errors in these

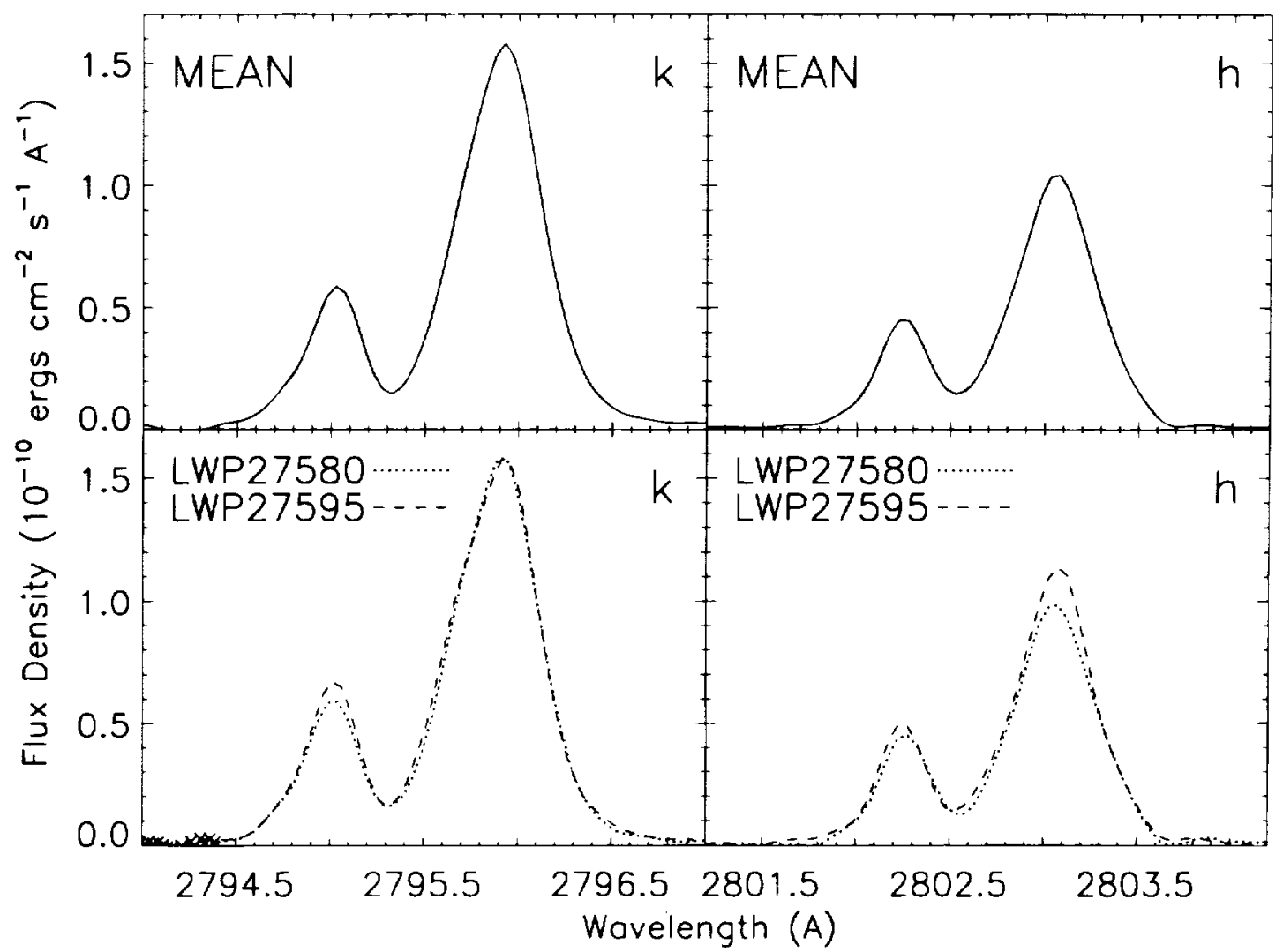

FIG. 3.-Profiles of $\mathrm{Mg}$ II $h$ and $k$ emission lines. Upper panel displays the time-averaged profiles calculated from the 23 short exposures in the $I U E$ time series. Lower panel displays the profiles obtained at the beginning and end of the series of observations made between JD $(+2,440,000) 9417.0$ and 9417.5 (see Fig. 2). 


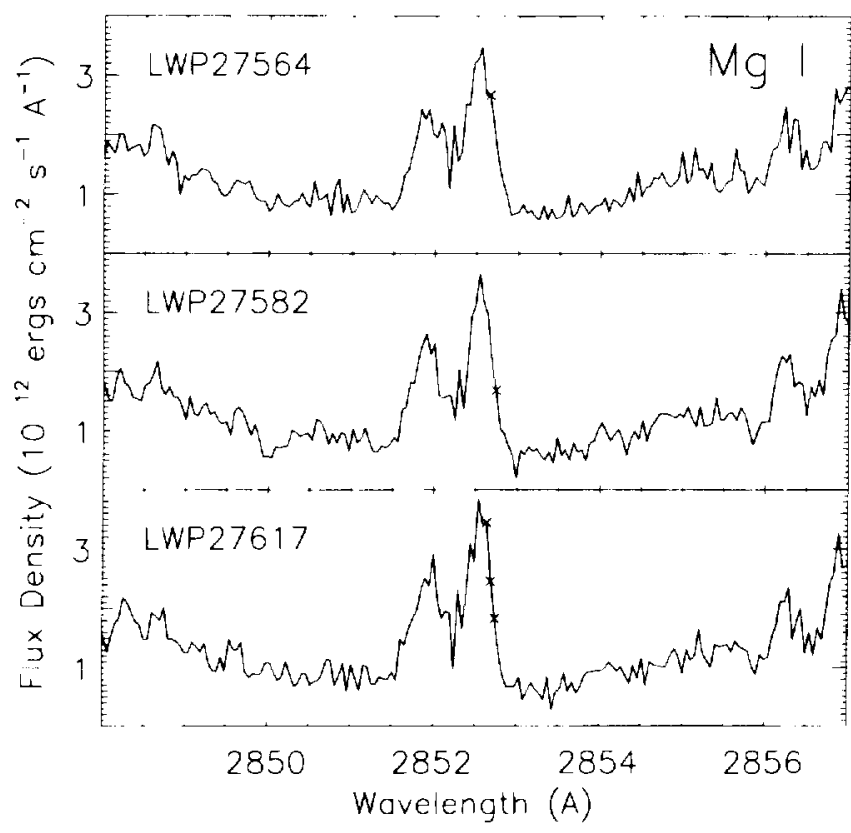

Fig. 4. - Line profiles for $\mathrm{Mg}$ I 22852 obtained from three 90 minute IUE exposures (LWP 27564, 27582, and 27617). Julian dates $(+2,440,000)$ at the beginning of the observations were $9416.007,9417.056$, and 9420.057 , respectively.

numbers are difficult to estimate because of the poor quality of the data and the blend by the (potentially time-variable) Fe II line, which prevent us to use $R_{2}$ and $R_{3}$ for additional electron density determinations. Therefore, we do not infer time variability from these data. The values are thus consistent with the results from previous studies of $\alpha$ Tau: Judge (1986) determined that $\log N_{e}=9.0 \pm 0.3$; Byrne et al. (1988) found $\log N_{e}=9.1 \pm 0.2$; and Carpenter et al. (1991), who calculated all three intensity ratios using HST GHRS spectra, deduced an electron density of slightly less than $\log N_{e}=9.0$. Preliminary results of another study, given by Stencel et al. (1992), also indicate electron densities of about $\log N_{e}=9.0$, commensurate with the other studies. Thus, we conclude that the $\mathrm{Mg}$ II variability does not appear to be

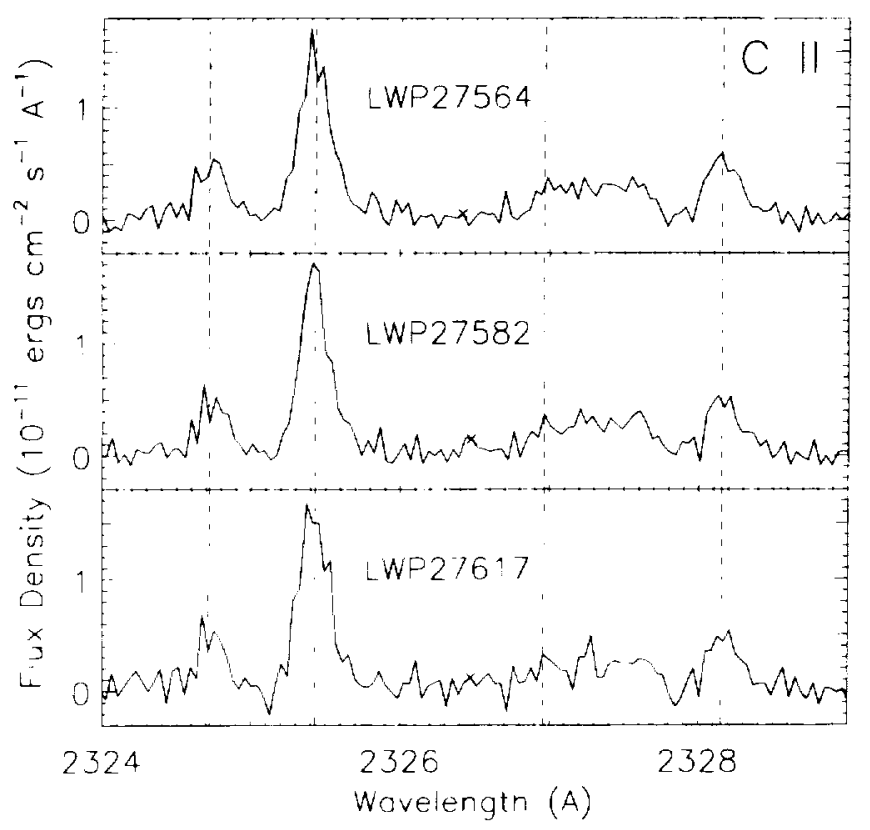

FIG. 5.- Same as Fig. 4, but now for the $\mathrm{C} \mathrm{II]} \lambda 2325$ multiplet associated with anomalous conditions in the chromosphere of $\alpha$ Tau and is probably a common occurrence.

\subsection{Comments on Theoretical Chromosphere Models}

Although initial chromospheric modeling was performed on the basis of time-independent theory (e.g., Hartmann \& MacGregor 1980), it now appears that chromospheric heating and mass loss in giant stars are greatly influenced by time-dependent phenomena. Cuntz (1987) studied the response of the outer atmosphere of $\alpha$ Boo (K1.5 III) to acoustic shocks generated in a "realistic" photospheric model (Bohn 1984). He found strong time-dependent episodes of momentum and energy deposition that control the chromospheric heating. Similar models were produced for additional stars and have been further improved. Cuntz (1990a) calculated theoretical model chromospheres for $\alpha$ Tau based on acoustic shock wave dissipation. These models do not consider the formation of distinct chromospheric lines but try to estimate the loss of radiative energy by employing a thin-plasma approximation. Such an approach is motivated by the work of Judge (1990), who showed that lines such as $\mathrm{Mg}$ II $k$ thermalize in the upper photospheres of inactive low-gravity stars. These lines are thus effectively thin in the entire chromosphere, and optically thin approximations (rather than more sophisticated techniques such as radiative transfer with partial redistribution) may be used in the calculation of the line cooling. The calculations of Cuntz (1990a) solve the hydrodynamic equations fully consistently, with the shocks treated as discontinuities.

An important aspect of these chromospheric models is the consideration of shock-shock interactions in determining the outer atmospheric dynamics. Shock-shock interactions are caused by height-dependent effects in the propagation of acoustic frequency spectra generated by stellar convection (Bohn 1984; Musielak et al. 1994; Kumar 1994). When acoustic wave modes propagate through the stellar atmosphere, shocks of different strengths and, therefore, different speeds are produced at different atmospheric heights. The range in shock speed is sufficiently broad to allow high-speed shocks to overtake, interact with, and engulf slower moving ones. Since an overtaking shock acquires the strength of the shock it engulfs, its speed increases, so that it can overtake more and more shocks in front of it. Consequently, the amount of momentum and energy deposition into the atmosphere varies drastically with height, depending on the atmospheric dynamics and the photospheric frequency spectrum. This scenario promotes the generation of distinct variability timescales for chromospheric lines in the case where only a limited number of strong shocks exists simultaneously in the atmosphere.

Figure 6 shows the expected distribution of chromospheric heating events in the chromosphere of $\alpha$ Tau at different atmospheric heights, as given by an acoustic heating model. Since the $\mathrm{Mg}$ II diagnostics form over roughly 4-5 orders of magnitude in mass column density, it is possible to compare the observed variability timescale with the estimates from the theoretical model. In the case of the $\mathrm{Mg}$ if $h$ and $k$ line wings, no variations were found. This is different for the $\mathrm{Mg}$ II line cores, which are formed around $10^{-4}$ and $10^{-5} \mathrm{~g} \mathrm{~cm}^{-1}$ mass column density. Figure 6 predicts a $\mathrm{Mg}$ II variability timescale of $2-8 \times 10^{4} \mathrm{~s}(5-20 \mathrm{hr})$ for the case in which the $\mathrm{Mg}$ II emission is associated with a 

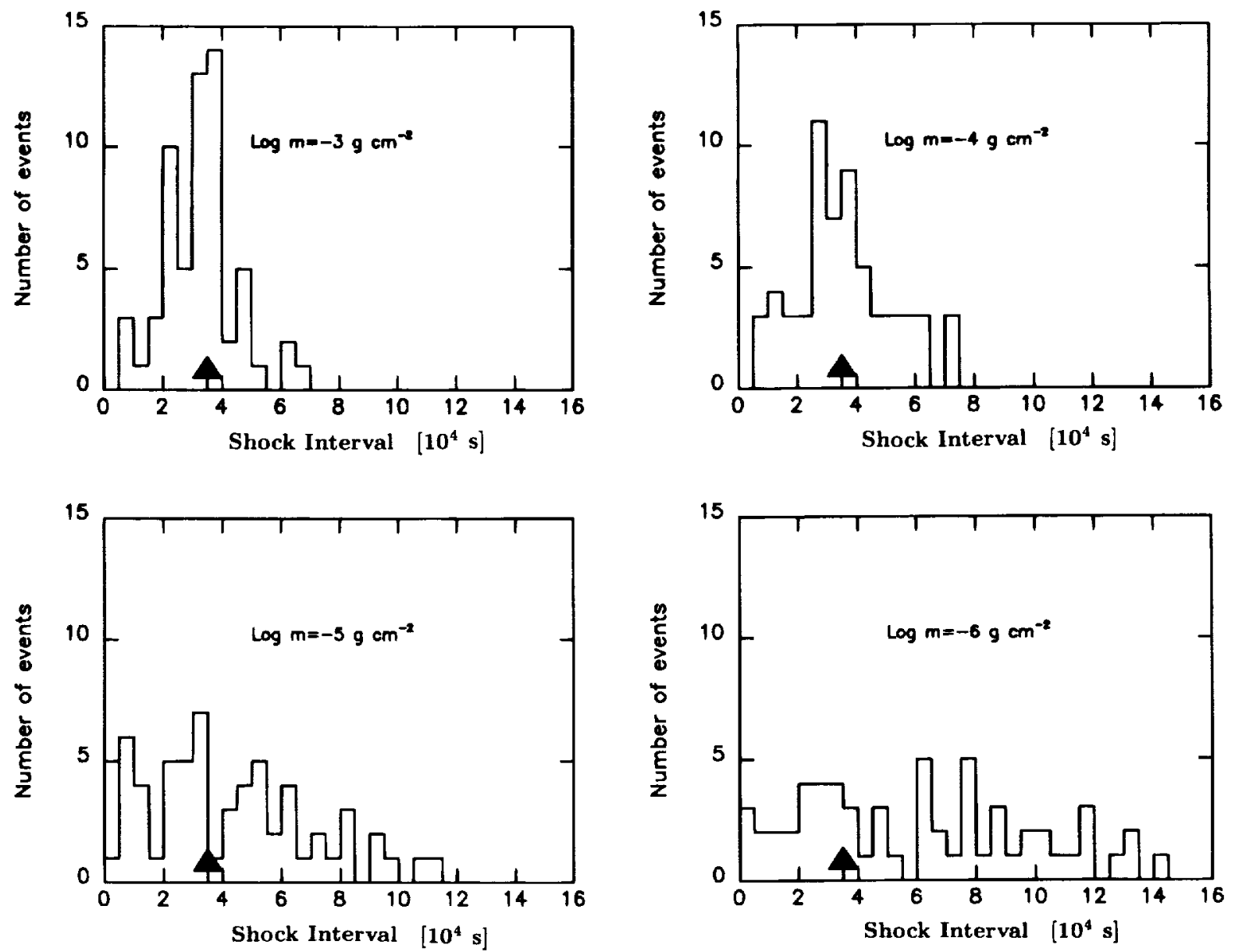

FiG. 6.-Histograms showing the number of shocks present in the time-dependent ab initio $\alpha$ Tau model chromosphere of Cuntz (1990a) as a function of the elapsed time between the shocks at a given mass column density. The input spectrum of waves was introduced at $2.5 \times 10^{-2} \mathrm{~g} \mathrm{~cm}-2$, with a mean period indicated by the arrow, and with a Gaussian width (FWHM) of $10^{4} \mathrm{~s}$. Periods are subject to stochastic changes, but systematically increase with height. Mg II lines are formed over regions from $10^{-2}$ (line wings) to $10^{-5} \mathrm{~g} \mathrm{~cm}^{-2}$ (line cores) mass column density.

small number of stochastic shocks. This timescale is similar to (or moderately larger than) the period in which the maximum of the acoustic frequency spectrum occurs, which has been introduced into the atmosphere.

The heating model used to produce Figure 6 assumes a photospheric energy spectrum centered at $3.5 \times 10^{4} \mathrm{~s}(=10$ $\mathrm{hr}$ ), consistent with traditional models of acoustic energy generation, as given by Bohn (1984). Bohn found that the maximum acoustic energy flux occurs at the driving period $P_{\max }=\epsilon P_{\mathrm{CO}}=4 \pi c_{z} \epsilon / \gamma g_{*}$, where $P_{\mathrm{CO}}$ is the acoustic cutoff period, $c_{z}$ is the sound speed at the top of the convection zone, $\gamma$ is the ratio of the specific heats, and $g_{*}$ is the stellar surface gravity. The parameter $\epsilon$, which denotes the position of the energy maximum in the acoustic frequency spectrum, is largely unconstrained due to uncertainties in the calculation of the initial acoustic energy spectrum from the model of stellar convection (see, e.g., Ulmschneider, Theurer, \& Musielak 1996).

Earlier work of Bohn (1984), as well as recent results of Ulmschneider et al. (1996), suggests that $\epsilon$ ranges between 0.10 and 0.30 for late-type giants. For the model given in Figure 6 , we took $\epsilon=0.10$. In the case that $\epsilon=0.30$ is adopted, the predicted $\mathrm{Mg}$ II variability timescale will change to $0.6-2.4 \times 10^{5} \mathrm{~s}(15-60 \mathrm{hr})$. This range comfortably encompasses the $\mathrm{Mg}$ II variability timescale seen in our observations. This prediction gives some confidence that the timescales of the observed variations are consistent with the presence of acoustic shocks propagating through the stellar atmosphere. The theoretically deduced distribution of the chromospheric heating events is also influenced by uncertainties in $g_{*}$. For the model given in Figure 6, we took $\log g_{*}=1.3$ (van Paradijs \& Meurs 1974; Judge \& Stencel 1991), which is uncertain by at least a factor of 2 . As a consequence, possible $P_{\max }$ are expected to spread over an even larger range than given above.

A further possible indication of the presence of shocks lies in the behavior of the $\mathrm{Mg} \mathrm{II} h / k$ ratio. It is found that the $\mathrm{Mg}$ II $h$ and $k$ fluxes are usually not correlated. It can be expected that the different optical depths of the two lines allow the change in emission to be detected first in the $\mathrm{Mg}$ II $h$ line while it is still too deep in the chromosphere to be seen in the $\mathrm{Mg}$ II $k$ line. These flux variations appear to be confined to the line core and thus occur in the outermost $\mathrm{Mg}$ II emitting regions. Changes in the $\mathrm{Mg}$ II $h / k$ ratio could thus indicate a shift of energy dissipation with atmospheric height, which is fully reconcilable with the behavior found in theoretical models of acoustic shock wave propagation.

\section{CONCLUSIONS}

The objective of this IUE observing program was to obtain new data with carefully arranged time spacing in order to examine the propagation of energy and momentum generated in subphotospheric convection zones through the atmosphere of $\alpha$ Tau. This program was aimed 
at gaining further insight into the mechanism(s) responsible for the observed atmospheric structures in cool, inactive giant stars. The star $\alpha$ Tau has often been considered a standard example of a giant with a noncoronal outer atmosphere, which is expected to be dominated by nonmagnetic heating. Global oscillations (if present) are irrelevant to the atmospheric gas dynamics in this type of star (Sutmann \& Cuntz 1995). The dominant factor in the dynamics and heating of such chromospheres should therefore be the propagation and dissipation of acoustic waves.

Our principal results can be summarized as follows:

1. We obtained $\mathrm{Mg}$ II observations with the IUE LWP camera at high dispersion in 1994 February-March. The observations show flux variations between the extremes of $8 \%$ in $\mathrm{Mg} \mathrm{II} h+k$ and $15 \%$ in the individual components. These variations occur on timescales as short as $8 \mathrm{hr}$. In one instance, we found a steady $6 \%$ increase in the $\mathrm{Mg}$ II $h+k$ flux occurring over a time span of 8.5-12 hr. Because of gaps in the data, the precise duration of this event cannot be determined. It can be said, however, that the $\mathrm{Mg}$ II flux increase did not persist for more than $\sim 3$ days. In another instance, we found evidence for a $5 \%$ increase occurring over a time span of 12-24 hr.

2. We found that the changes in $\mathrm{Mg}$ il $h$ and $k$ are often not correlated, leading to remarkable variations in the $h / k$ ratio. This ratio is found to vary between 0.60 and 0.70 . The uncorrelated flux variations can occur on timescales as short as $1.5 \mathrm{hr}$. It is possible that the different optical depths of the two lines allow a change in emission to be detected in the $h$ line while it is still too deep in the chromosphere to be seen in the $k$ line. The flux variations appear to be confined to the line core. Thus, they occur in the outermost $\mathrm{Mg}$ II emitting regions and might indicate spatial shifts in the atmospheric heating rate.

3. We deduced the electron density in the $\mathrm{C}$ II] $\lambda 2325$ line formation region at the epoch of our observations. We found electron densities of $\log N_{e} \simeq 9.0$, in agreement with results from Judge (1986), Byrne et al. (1988), and Carpenter et al. (1991).

4. We evaluated the level of chromospheric activity in $\alpha$ Tau based on the total $\mathrm{Mg}$ II emission flux. We found that the $\mathrm{Mg}$ Il surface flux agrees with those of other inactive $\mathrm{K}$ giants (see, e.g., Judge \& Stencel 1991). Our data indicate that the $\mathrm{Mg}$ II basal flux limit in $\mathrm{K}$ giants might be a factor of 4 higher than suggested by Rutten et al. (1991).

5. We argue that these results are consistent with the presence of strong acoustic shocks produced by the action of convective motions as the variability timescale of the $\mathrm{Mg}$ II flux is consistent with those expected from acoustic shock wave models. The shocks can lead to variable $\mathrm{Mg}$ II line emission when only a limited number of strong shocks exist simultaneously in the atmosphere and can account for the observed variations in the $\mathrm{Mg}$ II $h / k$ ratio.

We thank the IUE Observatory staff, particularly Don Luttermoser, for assistance in acquiring these data, and Jeffrey L. Linsky for valuable comments on an earlier version of the manuscript. M. C. acknowledges financial support through grant $\mathrm{Cu} 19 / 2-1$ provided by the German Research Foundation (DFG). This work was also supported by NASA grants NAG5-985 to the University of Colorado and NAG5-2103 to the University of Denver.
Ayres, T. R 1993, PASP 105,538

Ayres, T. R., et al. 1995, ApJS, 96, 223

Ayres, T. R., Fleming, T. A., \& Schmitt, J. H. M. M. 1991, ApJ, 376, L45

Bohn, H. U. 1984, A\&A, 136, 338

Brown, A., \& Carpenter, K. G. 1984, ApJ, 287, L43

Buchholz, B. 1995, Ph.D. thesis, Univ. Heidelberg

Buchholz, B., \& Ulmschneider, P. 1994, in ASP Conf. Ser. 64, Cool Stars,

Stellar Systems, and the Sun, ed. G. Wallerstein (San Francisco: ASP), 363

Byrne, P. B., Dufton, P. L., Kingston, A. E., Lennon, D. J., \& Murphy, H. M. 1988, ApJ, 197, 205

Carlsson, M., \& Stein, R. F. 1995, ApJ, 440, L29

Carpenter, K. G., Brown, A., \& Stencel, R. E. 1985, ApJ, 289, 676

Carpenter, K. G., Robinson, R. D., Wahlgren, G. M., Ake, T. B., Ebbets,

D. C. Linsky, J. L., Brown, A. \& Walter, F. M. 1991, ApJ 377 , L45

Cassatella, A., Lloyd, C., \& Gonzales Riestra, R. 1988, IUE NASA News, 35,225

Cassatella, A., Ponz, D., Selvelli, P. L., \& Vogel, M. 1990, IUE NASA News, 41, 155

Charbonneau, P., Schrijver, C. J., \& MacGregor, K. B. 1996, in Cosmic Winds and the Heliosphere, ed. J. R. Jokipii et al. (Tucson: Univ.

Arizona Press), in press

Cuntz, M. 1987, A\&A, 188, L5 .1990a, ApJ, 349, 141 . $1990 \mathrm{~b}$, ApJ, 353, 255

Cuntz M Rammacher W \& Ulmschneider, P 1994 ApJ 432,690

Di Benedetto, G. P., \& Rabbia, Y. 1987, A\&A, 188, 114

Dupree, A. K., Hartmann, L., \& Smith, G. H. 1990, ApJ, 353, 623

Garhart, M. P. 1992, IUE NASA News, 48, 80

Hartmann, L., \& Mac Gregor, K. B. 1980, ApJ, 242, 260

Hatzes, A. P., \& Cochran, W. D. 1993, ApJ, 413, 339

Jordan, C., \& Linsky, J. L. 1987, in Exploring the Universe with the IUE

Satellite, ed. Y. Kondo et al. (Dordrecht: Reidel), 259

Judge, P. G. 1986, MNRAS, 223, 239

\section{REFERENCES}

Judge, P. G. 1990, ApJ, 348, 279

1994, ApJ, 430, 351

Judge, P. G., \& Cuntz, M. 1993, ApJ, 409, 776

Judge, P. G., \& Jordan, C. 1991, ApJS, 77, 75

Judge, P. G., \& Stencel, R. E. 1991, ApJ, 371, 357

Kelch, W. L., Linsky, J. L., Basri, G. S., Chiu, H., Chang, S., Maram, S. P., \& Furenlid, I. 1978, ApJ, 220,962

Kumar, P. 1994, ApJ, 428, 827

Lennon, D. J., Dufton, P. L., Hibbert, A., \& Kingston, A. E. 1985, ApJ, 294. 200

Musielak, Z. E., Rosner, R., Stein, R. F., \& Ulmschneider, P. 1994, ApJ. 423,474

O'Brien, G. T. , \& Lambert, D. L. 1986, ApJS, 62, 899

Pasquini, L., Pallavicini, R., \& Pakull, M. 1988, A\&A, 191, 253

Rosner, R., An, C.-H., Musielak, Z. E., Moore, R. L., \& Suess, S. T. 1991, ApJ, 372, L91

Rosner, R., Musielak, Z. E., Cattaneo, F., Moore, R. L., \& Suess, S. T. 1995, ApJ, 442, L25

Rutten, R. G. M., Schrijver, C. J., Lemmens, A. F. P., \& Zwaan, C. 1991, A\&A, 252, 203

Schrijver, C. J. 1987, A\&A, 172, 111

- 1993 in ASP Conf. Ser. 40, Inside the Stars, ed. W. W. Weiss \& A. Baglin (San Francisco: ASP), 328

Stencel, R. E., Brown, A., Carpenter, K. G., Cuntz, M., \& Judge, P. G. 1992, BAAS, 24, 1175

Stencel, R. E., Linsky, J. L., Brown, A., Jordan, C., Carpenter, K. G., Wing. R. F., \& Czyzak, S. 1981, MNRAS, 196, 47P

Strassmeier, K. G., Handler, G., Paunzen, E., \& Rauth, M. 1994, A\&A. 281, 855

Sutmann, G., \& Cuntz, M. 1995, ApJ, 442, L61

Ulmschneider, P., Theurer, J., \& Musielak, Z. E. 1996, A\& A, submitted

van Paradijs, J., \& Meurs, E. J. A. 1974, A\& \&, 35, 225

Wiedemann, G., Ayres, T. R., Jennings, D. E., \& Saar, S. H. 1994, ApJ, 423. 806 
